In vivo analysis of the Mesenchymal-to-Epithelial transition during chick secondary neurulation

Elena Gonzalez-Gobartt ${ }^{1}$, Guillaume Allio ${ }^{2}$, Bertrand Bénazéraf ${ }^{2}$ and Elisa Martí ${ }^{1}$ 1-Instituto de Biología Molecular de Barcelona, CSIC, Parc Científic de Barcelona, C/Baldiri i Reixac 20, Barcelona 08028, Spain.

2- Centre de Biologie du Développement (CBD), Centre de Biologie Intégrative (CBI), Université de Toulouse, CNRS, UPS, Toulouse, France.

Corresponding author's name and e-mail: Elisa Martí - emgbmc@ibmb.csic.es

Running head: In vivo analysis of the MET during secondary neurulation 


\title{
In vivo analysis of the Mesenchymal-to-Epithelial transition during chick secondary neurulation
}

\author{
Elena Gonzalez-Gobartt ${ }^{1}$, Guillaume Allio ${ }^{2}$, Bertrand Bénazéraf ${ }^{2}$ and Elisa Martí ${ }^{1}$ \\ 1-Instituto de Biología Molecular de Barcelona, CSIC, Parc Científic de Barcelona, \\ C/Baldiri i Reixac 20, Barcelona 08028, Spain. \\ 2- Centre de Biologie du Développement (CBD), Centre de Biologie Intégrative (CBI), \\ Université de Toulouse, CNRS, UPS, Toulouse, France.
}

\begin{abstract}
The neural tube in amniote embryos forms as a result of two consecutive events along the antero-posterior axis, referred to as primary and secondary neurulation (PN and $\mathrm{SN}$ ). While PN involves the invagination of a sheet of epithelial cells, SN shapes the caudal neural tube through the mesenchymal-to-epithelial transition (MET) of neuromesodermal progenitor cells, followed by cavitation of the medullary chord. The technical difficulties in studying SN mainly involve the challenge of labelling and manipulating SN cells in vivo. Here we describe a new method to follow MET during $\mathrm{SN}$ in the chick embryo, combining early in ovo chick electroporation with in vivo timelapse imaging. This procedure allows the cells undergoing SN to be manipulated in order to investigate the MET process, permitting their cell dynamics to be followed in vivo.
\end{abstract}

Keywords: Mesenchymal-to-epithelial transition, Secondary neurulation, Neural tube formation, Chick embryo, In ovo electroporation, In vivo time-lapse imaging, Cell dynamics. 


\section{Introduction}

During development, the entire vertebrate peripheral nervous system (PNS) is formed through the epithelial-to-mesenchymal transition (EMT) of neuroepithelial cells, which generates migratory neural crest cells, one of the best studied examples of physiological EMT [1-3]. By contrast, the mesenchymal-to-epithelial transition (MET) is the reverse process and it plays an important role during organogenesis, as well as in the elongation of the caudal nervous system, a process known as secondary neurulation ( $\mathrm{SN})$. Indeed, the formation of the vertebrate neural tube (NT) involves two different morphogenetic events, primary $(\mathrm{PN})$ and secondary neurulation $(\mathrm{SN})[4,5]$. During PN, the lateral ends of the anterior neural plate elevate and the bilateral neural folds fuse with each other to form the anterior NT [6-8]. By contrast, in SN mesenchymal neuro-mesodermal progenitor cells are recruited to elongate the caudal body axis and drive the caudal elongation of the NT. The MET of neuro-mesodermal progenitor cells (NMPCs) is the central event of SN, along with the formation of a compact nerve cord and its subsequent cavitation to form the caudal NT. In human embryos, the transition from the primary to the secondary NT occurs at the lumbosacral level; therefore the development of the lumbar, sacral, coccygeal and equinal cord largely involves SN [9-12].

Here, we propose that the chick embryo is an ideal model to understand the MET events that occur during amniote SN. While manipulating and imaging mammalian embryos is still problematic, the chick embryo provides an easily accessible model in which the stages of development can be readily identified [13]. At early stages of development the avian body plan is very similar to that of mammals, and these embryos have excellent optical properties as they are thin and planar [14]. Furthermore, the SN region in the chick embryo extends up to the lumbar region $[15,16]$, closely resembling human development, whereas SN only occurs at the tail level in mice [17-19]. 
In the chick, SN starts when undifferentiated NMPCs converge onto the dorsal midline, adopt a neural cell identity and undergo MET. The complete MET process can be followed in stage HH15 chick embryos, as different degrees of polarisation exist along the anteroposterior axis (FIG. 1). The first cells to undergo MET are located in the periphery of the medullary cord, while the central cells remain mesenchymal until the very end of the process. It is between these two cell populations that small cavities of varied size and shape form, later coalescing to form a single central lumen (FIG. 1C-G) $[20,21]$.

However, the analysis of SN in vivo has always been technically difficult. Cell tracing studies have identified the epiblast region occupied by cells that undergo $\mathrm{SN}$ in the future (the preSN region), located caudo-medially to Hensen's Node at stage HH9 chick embryos [22-26]. However, the NT is still open in the posterior domains of stage HH9 chick embryos, so for electroporation the DNA must be injected on top of the epiblast and the electrodes positioned above and below the embryo (FIG. 2A-B). Earlier embryonic stages have always been electroporated ex ovo in this way $[27,28]$, which facilitates the electroporation of the flat epiblast, although cultured embryos do not grow to stage HH15 with normal body elongation [29].

Here we propose a new method for the in vivo analysis of MET during SN that overcomes all these technical difficulties. The method involves combining early in ovo chick embryo electroporation with time-lapse imaging in a culture set-up specifically adapted to avian embryos $[30,31,14]$. This technique allows any cells undergoing SN to be manipulated in vivo in order to investigate the MET process, and it permits cell dynamics to be followed in vivo. Briefly, stage HH9 chick embryos are electroporated in ovo to transform the preSN region, injecting the DNA onto the surface of the concave cavity that exists at the posterior end of the embryo, where the primary NT is still open. 
The electrodes are then positioned carefully above and below the embryo (FIG. 2A-B), and an electrical current is applied so that the posterior cells incorporate the plasmid DNA. Subsequently, the eggs are sealed and incubated for $24 \mathrm{~h}$, until the embryos reach stage HH15 (FIG. 2D-G). The next day the eggs are opened again and the embryos are removed using a filter paper ring (FIG. 3A-B), which not only facilitates their manipulation but also provides the mechanical support essential to generate the correct tensions and deformation that occur during normal embryo elongation [29]. Embryos attached to these rings are then cleaned to avoid any yolk and debris interfering with their visualization, and they are transferred to pre-prepared imaging plates containing Agar/Albumen culture media (FIG. 3C). Finally, the embryos are placed in a culture chamber (FIG. 3D-E) and examined under an upright wide-field microscope. The system we describe here allows 6 specimens to be visualized simultaneously and they can be analysed over long periods of time, these embryos developing at approximately the same rate as they do in ovo (FIG. 4, MOVIE S1). In the videos generated with this system, the cells undergoing MET can be visualised and tracked during the process of SN (FIG. 5, MOVIE S2-S3).

\section{Materials}

\subsection{Electroporation of chick $\mathrm{SN}$ cells in ovo}

1. Fertilized eggs are obtained from the White-Leghorn chicken strain.

2. Electroporation mixture containing the plasmid DNA. Here we use plasmids containing pSox2:eGFP [32-34] and the TopFlash:d2eGFP $[35,36]$ reporters as examples. Before injection, the DNA plasmids are diluted to $2 \mu \mathrm{g} / \mu \mathrm{l}$ in $60 \%$ sucrose prepared in $\mathrm{H}_{2} \mathrm{O}$, adding 1:10 of Fast Green FCF. 
3. TSS-20 Ovodyne Electroporator operated by a footswitch or equivalent equipment generating square electrical pulses.

4. Electrodes (FIG. 2C). We separated a pair of commercial platinum electrodes (CUY610P1.5-1, Nepagene or equivalent) and only used one side as the positive electrode. We incorporated a sharpened and bent $90^{\circ}$ tungsten needle (Fine Science Tools) into a holder and used it as the negative 'microelectrode' $[37,38]$.

5. Glass capillaries with a filament (GD-1, Narishige or equivalent) are used to make injection needles with a glass capillary puller (PC-10, Narishige or equivalent).

6. Aspirator tube assemblies for calibrated microcapillary pipettes (Sigma-Aldrich, A5177-5EA or equivalent).

7. $1 \%$ Penicillin/Streptomycin (P/S) in MilliQ $\mathrm{H}_{2} \mathrm{O}$.

8. Syringe, thin forceps, curved scissors and plastic tape.

\subsection{Imaging plate preparation}

1. Millicell cell culture plate inserts (0.4 mm: Millipore, PICMORG50).

2. $25 \mathrm{~mL}$ of thin albumen from eggs incubated overnight.

3. $10 \%$ glucose in MilliQ $\mathrm{H}_{2} \mathrm{O}$.

4. $0.6 \%$ Granulated Agar (Difco, 0145-17-0) in MilliQ $\mathrm{H}_{2} \mathrm{O}$.

5. $5 \mathrm{M} \mathrm{NaCl}$ in MilliQ $\mathrm{H}_{2} \mathrm{O}$.

6. $\mathrm{P} / \mathrm{S}$, undiluted.

7. Water bath, $50 \mathrm{~mL}$ Falcon tubes and Pasteur pipettes.

\subsection{Mounting and in vivo time-lapse imaging}

1. Filter paper rings prepared from $2 \times 2 \mathrm{~cm}$ squares of Whatman grade 1 filter paper in which a clover-leaf shaped hole is made in the centre with a paper punch, cutting the corners so that they fit in the round imaging plates (FIG. 3A). 
2. PBS $1 \times(1 \mathrm{~L}): 8 \mathrm{~g} \mathrm{NaCl}, 0.2 \mathrm{~g} \mathrm{KCl}, 1.44 \mathrm{~g} \mathrm{Na}_{2} \mathrm{HPO}_{4}, 0.24 \mathrm{~g} \mathrm{KH}_{2} \mathrm{PO}_{4}, 800 \mathrm{~mL}$ of MilliQ $\mathrm{H}_{2} \mathrm{O}$, adjusted $\mathrm{pH}$ to 7.4 and to $1 \mathrm{~L}$, sterilized by autoclaving and stored at room temperature.

3. $5 \mathrm{~mL}$ of thin albumen from eggs incubated overnight.

4. $5 \mathrm{~mL}$ of $123 \mathrm{mM} \mathrm{NaCl}$ in MilliQ $\mathrm{H}_{2} \mathrm{O}$.

5. Soft tissues (Kimtech Science Kimwipes, or equivalent), thin forceps, fine scissors, Pasteur pipettes, electrical insulation tape and $100 \mathrm{~mm}$ petri dishes.

6. Culture chamber [14] created from a Corning ${ }^{\circledR}$ Costar ${ }^{\circledR}$ polystyrene 6-well plate (Sigma, CLS3736 or equivalent). To favour the optics, the plastic in the lid is replaced with glass. In each well, a $23 \mathrm{~mm}$ hole is made in the centre of the lid by pushing a heated cork borer through the plastic, smoothing the rough edges and sealing a $25 \mathrm{~mm}$ diameter glass \#1 coverslip over the hole using Marine Adhesive (Zolux Silicone "SA 500”) (FIG.3D).

\subsection{Image processing and analysis}

1. ZEN software (Zeiss) Version 2.3 blue edition with the experiment designer option or any equivalent option allowing time-lapse imaging with multiple XYZ positions.

2. Image J/Fiji software $[39,40]$.

3. BioFormat plugin [41] (https://imagej.net/Bio-Formats).

4. Image5D (Joachim Walter - https://imagej.nih.gov/ij/plugins/image5d.html) plugins.

5. Stack focuser (Michael Umorin - https://imagej.nih.gov/ij/plugins/stackfocuser.html) plugin.

6. MultiStackReg plugin (Brad Busse and Kota Miura http://bradbusse.net/downloads.html). 
7. StackReg plugin [42] (http://bigwww.epfl.ch/thevenaz/stackreg/).

8. Grid/Collection stitching plugin [43] (https://imagej.net/Image_Stitching\#Grid.2FCollection_Stitching).

9. Manual Tracking plugin (Fabrice P. Cordelières https://imagej.nih.gov/ij/plugins/track/track.html).

\section{Methods}

\subsection{Electroporation of chick $\mathrm{SN}$ cells in ovo}

1. Incubate eggs horizontally at $38.5^{\circ} \mathrm{C}$ in an atmosphere of $70 \%$ humidity until stage HH9.

2. Remove $5 \mathrm{~mL}$ of albumen from the egg with a syringe.

3. Open a window at the top of the shell with curved scissors to visualize the embryo.

4. Make a small hole with thin forceps in the posterior region of the area opaca, just outside of the area pellucida. Avoid touching the embryo (FIG. 2A).

5. Pour $200 \mathrm{~mL}$ of $1 \% \mathrm{P} / \mathrm{S}$ onto the embryo to improve electrode conductivity (see Note $1)$.

6. Inject the DNA solution onto the epiblast with a glass capillary by blowing air through the aspirator tube. Introduce the DNA into the small concave region at their posterior end of the stage $\mathrm{HH} 9$ embryo, where the neural tube is still open (FIG. 2A-B: see Notes $2 \& 3)$.

7. Carefully insert the platinum electrode connected to the positive lead (+) below the embryo through the hole made previously, parallel to its antero-posterior axis (FIG. 2AB).

8. Position the tungsten microelectrode connected to the negative lead (-) on top of the embryo, also parallel to the embryo's antero-posterior axis (FIG. 2A-B). 
9. Deliver five $50 \mathrm{~ms}$ square pulses of $5 \mathrm{~V}$ at intervals of $50 \mathrm{~ms}$ with the electroporator (see Note 4).

10. Seal the window in the shell with tape and incubate embryos until they reach stage HH15 (+24h).

\subsection{Imaging plate preparation}

1. Heat a water bath to $50{ }^{\circ} \mathrm{C}$. Collect $25 \mathrm{~mL}$ of thin albumen from the eggs incubated overnight in a sterile $50 \mathrm{~mL}$ tube and stir it for $15 \mathrm{mins}$ at RT (see Note 5).

2. Add $0.75 \mathrm{~mL}$ of $10 \%$ glucose to the albumen and place the mixture in the water bath.

3. Boil $25 \mathrm{~mL}$ of $0.6 \%$ BactAgar in MilliQ $\mathrm{H}_{2} \mathrm{O}$ and add $0.615 \mathrm{~mL}$ of $5 \mathrm{M} \mathrm{NaCl}$.

Transfer this solution into another sterile $50 \mathrm{~mL}$ tube and place it into the water bath to equilibrate to $50^{\circ} \mathrm{C}$.

4. Mix the solutions of Albumen/Glucose and Agar/ $\mathrm{NaCl}$ in a sterile $50 \mathrm{~mL}$ tube and add $100 \mu \mathrm{l}$ of $\mathrm{P} / \mathrm{S}$ (see Note 6 ).

5. Use a sterile Pasteur pipette to pour $1.5 \mathrm{~mL}$ of the Albumen/Agar mix onto the cell culture inserts on a flat surface (see Note 7), leaving the Albumen/Agar imaging plates at RT to cool until the gel becomes solid and then storing them at $4{ }^{\circ} \mathrm{C}$ (see Note 8).

\subsection{Mounting and in vivo time-lapse imaging}

1. Reopen the tape-sealed window in the egg, and carefully remove the thick albumen surrounding and covering the embryo with a soft tissue (see Note 9).

2. Place a paper ring on top of the vitelline membrane so that the embryo is located in the center of the clover-shaped hole (FIG. 3A-B), and cut through the vitelline membrane and around the whole perimeter of the filter paper ring with a small pair of scissors, carefully pulling the filter with the embryo attached away from the yolk (see Note 10). 
3. Place the embryo ventral side up in a petri dish containing 1x PBS, and clean the remaining yolk and debris by blowing streams of PBS over the embryo with a Pasteur pipette (see Note 11).

4. Select the embryos with the best overall morphology and the greatest level of transgene expression for imaging (see Note 12).

5. Transfer the selected embryos to an Agar/Albumen imaging plate dorsal side up (FIG. 3C), and fill each well of the culture chamber with $1.5 \mathrm{~mL}$ of a solution of $5 \mathrm{ml}$ thin albumen and $5 \mathrm{ml}$ of $123 \mathrm{mM} \mathrm{NaCl}$.

6. Transfer the embryos in the imaging plates to the wells of the culture chamber (see Note 13) and add 1x PBS between the wells to maintain a moist environment inside the culture chamber. Seal the culture chamber with electrical insulation tape so that up to 6 embryos can be imaged at the same time (FIG. 3D-E: see Note 14).

7. Visualize the embryos under an upright wide-field microscope Axio Imager 2 (Zeiss) equipped with a motorized stage and an incubation chamber. Set the temperature to 39.5 ${ }^{\circ} \mathrm{C}$ so that the temperature at the level of the embryo is around $37.5^{\circ} \mathrm{C}$ (see Note 15 ). 8. Use the Experiment designer module of the ZEN software to set up the acquisition parameters, creating one experimental block for each embryo (i.e.: one 6-well plate $=6$ blocks). Define the acquisition parameters for each block after focusing and positioning the embryo in the centre of the field of view (see Note 16).

9. Define a last "blank" block situated in the middle of the imaging plate with the objective in a higher position.

10. Create a "delay block" as the final block and define the delay, which will be the time interval in the time-lapse movie. This delay is synchronised with the preceding blocks and it can be used to pause the process in order to correct drift or loss of focus (see Note 17). 
11. Define the number of acquisition loops, which will correspond to the number of time points in the time-lapse video. The motorized stage is now set automatically to move to each previously defined block position, where the given numbers of tiles, channels and slices will be acquired, a process that will be repeated for each loop in every given time interval.

12. With a $5 \mathrm{x}$ objective, acquire $10 \mathrm{z}$ images every 10 minutes at a resolution of $1024 \mathrm{x}$ 1024, binning 4 x 4 (FIG. 4, MOVIE S1). With a 20x objective, acquire $10 \mathrm{z}$ images every 6 minutes at a resolution of 1024 x 1024, binning 4 x 4 (FIG. 5: see Note 18).

\subsection{Image processing and analysis}

1. Time stitch the images of each embryo acquired with the ZEN software.

2. Open the Tiff file in ImageJ/Fiji using the BioFormat Importer plugin.

3. Convert each tile to the Image5D format (In ImageJ: Plugin tab/Image5D/Stack to Image 5D) and apply the Stack focuser plugin, which allows the best focused parts to be selected and projected onto the same plane (see Note 19). Then convert the best focused projection in the Image5D format to the hyperstack format (In ImageJ: Plugin tab/Image5D/Image5D to stack and then Image tab/Hyperstack/Stack to Hyperstack and redefine the dimensions). Repeat the process for each time point of the time-lapse.

4. Stitch together the best focused tiles using the Grid/Collection stitching plugin in order to reconstruct the whole best focused time-lapse movie.

5. Finally, correct the drift along the time-lapse video using the MultiStackReg plugin that is based on the StackReg plugin (see Note 20). The best focused time-lapse movie is now aligned (FIG. 5A-B, MOVIE S2).

6. Individual cells are followed spatiotemporally using the Manual Tracking plugin of the Image J software (FIG. 5C-D, MOVIE S3).

\section{Notes}


1. It is best to perform steps 4 and 5 before placing the electroporation mixture on the epiblast to avoid its diffusion.

2. Repeated injection of DNA through the membranes may block the needle, in which case, clean the tip with a wet tissue or cotton swab.

3. The electroporation mixture can be passed over the top of the epiblast by blowing air through the aspirator tube or with the help of a syringe.

4. Maintain the electrodes moist with $1 \%$ of $\mathrm{P} / \mathrm{S}$ in MilliQ $\mathrm{H}_{2} \mathrm{O}$ to diminish the resistance between the electrodes.

5. To prepare the imaging plates, collect the thin albumen at the time of electroporation with a syringe, as both procedures are performed on the same day. Thin albumen can also be collected by cracking an egg incubated overnight into a petri dish and recovering it with a Pasteur pipette.

6. Wait for the two solutions to equilibrate to the same temperature as the hot agar could "cook" the thin albumen if they are mixed too early.

7. Try to fill the culture inserts in a way that the culture medium dries as flat as possible, avoiding the introduction of bubbles.

8. Imaging plates should be prepared fresh, no more than 2 days in advance. The plates can be stored at $4{ }^{\circ} \mathrm{C}$ in sterile $35 \mathrm{~mm}$ petri dishes.

9. The albumen prevents the membranes from attaching to the filter paper so try to remove as much as possible. However, if the embryo is already dry and it is touched again with the tissue, the blastoderm may attach and break and the embryo will be lost. 10. Pull the filter paper with the embryo away from the yolk obliquely. It is best to pull in the direction of the yolk flow produced by the cutting of membranes or along the anteroposterior embryonic axis. 
11. Do not blow streams of PBS directly onto the embryo. Clean it by blowing away from the centre where the embryo is located, towards the sides of the filter paper.

12. Electroporated embryos can be screened and selected in the egg, in PBS or in $35 \mathrm{~mm}$ petri dishes embedded in the Agar/Albumen mix. These can be done when preparing the imaging plates.

13. Dip the bottom of the plates in PBS before transferring them to the culture chamber to avoid forming bubbles under the imaging plates.

14. Before sealing the culture chamber and to avoid any condensation during image acquisition, the coverslip should be sprayed with an antifog solution typically used for scuba diving masks.

15. Place the 6-well plate into the incubation chamber of the microscope but do not start the video recording straight away. Leave the embryos to recover for at least $1 \mathrm{~h}$.

16. Define the number of slices, number of tiles, number of channels, laser power and exposure time. Images are acquired in a mosaic format, with different tiles representing each field of view.

17. Frequently check the time-lapse image acquisition during the first hours of imaging as the focus may be lost due to the embryo settling down and due to its elongation.

Refocus as often as necessary, during the pausing time.

18. In vivo time-lapse imaging of $\mathrm{SN}$ cells can be performed using other systems using some mounting modifications. For example, for confocal imaging we mount the embryos in Lab-Tek ${ }^{\circledR}$ 2-well glass chamber slides.

19. The Stack focuser plugin detects the sharpest details of each image in a z-stack.

20. Registration is calculated on the channel displaying the highest contrast and it is then applied to the other channels. 


\section{References}

1. Gouignard N, Andrieu C, Theveneau E (2018) Neural crest delamination and migration: Looking forward to the next 150 years. Genesis 56 (6-7):e23107. doi:10.1002/dvg.23107

2. Mayor R, Theveneau E (2013) The neural crest. Development 140 (11):2247-2251. doi:10.1242/dev.091751

3. Theveneau E, Mayor R (2012) Neural crest migration: interplay between chemorepellents, chemoattractants, contact inhibition, epithelial-mesenchymal transition, and collective cell migration. Wiley Interdiscip Rev Dev Biol 1 (3):435-445. doi:10.1002/wdev.28

4. Harrington MJ, Hong E, Brewster R (2009) Comparative analysis of neurulation: first impressions do not count. Mol Reprod Dev 76 (10):954-965. doi:10.1002/mrd.21085 5. Lowery LA, Sive H (2004) Strategies of vertebrate neurulation and a re-evaluation of teleost neural tube formation. Mech Dev 121 (10):1189-1197.

doi:10.1016/j.mod.2004.04.022

6. Smith JL, Schoenwolf GC (1987) Cell cycle and neuroepithelial cell shape during bending of the chick neural plate. Anat Rec 218 (2):196-206.

doi:10.1002/ar.1092180215

7. Colas JF, Schoenwolf GC (2001) Towards a cellular and molecular understanding of neurulation. Developmental dynamics : an official publication of the American Association of Anatomists 221 (2):117-145. doi:10.1002/dvdy.1144 
8. Nikolopoulou E, Galea GL, Rolo A, Greene ND, Copp AJ (2017) Neural tube closure: cellular, molecular and biomechanical mechanisms. Development 144 (4):552566. doi:10.1242/dev.145904

9. Saitsu H, Shiota K (2008) Involvement of the axially condensed tail bud mesenchyme in normal and abnormal human posterior neural tube development. Congenit Anom (Kyoto) 48 (1):1-6. doi:10.1111/j.1741-4520.2007.00178.x 10. Saitsu H, Yamada S, Uwabe C, Ishibashi M, Shiota K (2004) Development of the posterior neural tube in human embryos. Anat Embryol (Berl) 209 (2):107-117. doi:10.1007/s00429-004-0421-2

11. O'Rahilly R, Muller F (2002) The two sites of fusion of the neural folds and the two neuropores in the human embryo. Teratology 65 (4):162-170. doi:10.1002/tera.10007 12. O'Rahilly R, Muller F (1994) Neurulation in the normal human embryo. Ciba Found Symp 181:70-82; discussion 82-79

13. Hamburger V, Hamilton HL (1992) A series of normal stages in the development of the chick embryo. 1951. Developmental dynamics : an official publication of the American Association of Anatomists 195 (4):231-272. doi:10.1002/aja.1001950404 14. Rupp PA, Rongish BJ, Czirok A, Little CD (2003) Culturing of avian embryos for time-lapse imaging. BioTechniques 34 (2):274-278. doi:10.2144/03342st01 15. Dady A, Havis E, Escriou V, Catala M, Duband JL (2014) Junctional neurulation: a unique developmental program shaping a discrete region of the spinal cord highly susceptible to neural tube defects. J Neurosci 34 (39):13208-13221. doi:10.1523/JNEUROSCI.1850-14.2014

16. Criley BB (1969) Analysis of embryonic sources and mechanims of development of posterior levels of chick neural tubes. J Morphol 128 (4):465-501. doi:10.1002/jmor.1051280406 
17. Shum AS, Tang LS, Copp AJ, Roelink H (2010) Lack of motor neuron differentiation is an intrinsic property of the mouse secondary neural tube. Developmental dynamics : an official publication of the American Association of Anatomists 239 (12):3192-3203. doi:10.1002/dvdy.22457 18. Schoenwolf GC (1984) Histological and ultrastructural studies of secondary neurulation in mouse embryos. Am J Anat 169 (4):361-376. doi:10.1002/aja.1001690402 19. Nievelstein RA, Hartwig NG, Vermeij-Keers C, Valk J (1993) Embryonic development of the mammalian caudal neural tube. Teratology 48 (1):21-31. doi:10.1002/tera.1420480106 20. Schoenwolf GC, Delongo J (1980) Ultrastructure of secondary neurulation in the chick embryo. Am J Anat 158 (1):43-63. doi:10.1002/aja.1001580106

21. Schoenwolf GC, Kelley RO (1980) Characterization of intercellular junctions in the caudal portion of the developing neural tube of the chick embryo. Am J Anat 158 (1):29-41. doi:10.1002/aja.1001580105

22. Shimokita E, Takahashi Y (2011) Secondary neurulation: Fate-mapping and gene manipulation of the neural tube in tail bud. Dev Growth Differ 53 (3):401-410. doi:10.1111/j.1440-169X.2011.01260.x

23. Catala M, Teillet MA, De Robertis EM, Le Douarin ML (1996) A spinal cord fate map in the avian embryo: while regressing, Hensen's node lays down the notochord and floor plate thus joining the spinal cord lateral walls. Development 122 (9):2599-2610 24. Catala M, Teillet MA, Le Douarin NM (1995) Organization and development of the tail bud analyzed with the quail-chick chimaera system. Mech Dev 51 (1):51-65 25. Le Douarin NM, Teillet MA, Catala M (1998) Neurulation in amniote vertebrates: a novel view deduced from the use of quail-chick chimeras. Int J Dev Biol 42 (7):909-916 
26. Le Douarin NM (2001) Early neurogenesis in Amniote vertebrates. Int J Dev Biol $45(1): 373-378$

27. Voiculescu O, Papanayotou C, Stern CD (2008) Spatially and temporally controlled electroporation of early chick embryos. Nat Protoc 3 (3):419-426.

doi:10.1038/nprot.2008.10

28. Hatakeyama J, Shimamura K (2008) Method for electroporation for the early chick embryo. Dev Growth Differ 50 (6):449-452. doi:10.1111/j.1440-169X.2008.01040.x 29. Chapman SC, Collignon J, Schoenwolf GC, Lumsden A (2001) Improved method for chick whole-embryo culture using a filter paper carrier. Developmental dynamics : an official publication of the American Association of Anatomists 220 (3):284-289. doi:10.1002/1097-0177(20010301)220:3<284::AID-DVDY1102>3.0.CO;2-5

30. Benazeraf B, Beaupeux M, Tchernookov M, Wallingford A, Salisbury T, Shirtz A, Shirtz A, Huss D, Pourquie O, Francois P, Lansford R (2017) Multi-scale quantification of tissue behavior during amniote embryo axis elongation. Development. doi:10.1242/dev.150557

31. Benazeraf B, Francois P, Baker RE, Denans N, Little CD, Pourquie O (2010) A random cell motility gradient downstream of FGF controls elongation of an amniote embryo. Nature 466 (7303):248-252. doi:10.1038/nature09151

32. Saade M, Gutierrez-Vallejo I, Le Dreau G, Rabadan MA, Miguez DG, Buceta J, Marti E (2013) Sonic hedgehog signaling switches the mode of division in the developing nervous system. Cell Rep 4 (3):492-503. doi:10.1016/j.celrep.2013.06.038 33. Uchikawa M, Ishida Y, Takemoto T, Kamachi Y, Kondoh H (2003) Functional analysis of chicken Sox 2 enhancers highlights an array of diverse regulatory elements that are conserved in mammals. Dev Cell 4 (4):509-519 
34. Le Dreau G, Saade M, Gutierrez-Vallejo I, Marti E (2014) The strength of

SMAD1/5 activity determines the mode of stem cell division in the developing spinal cord. J Cell Biol 204 (4):591-605. doi:10.1083/jcb.201307031

35. Rios AC, Denans N, Marcelle C (2010) Real-time observation of Wnt beta-catenin signaling in the chick embryo. Developmental dynamics : an official publication of the American Association of Anatomists 239 (1):346-353. doi:10.1002/dvdy.22174

36. Serralbo O, Marcelle C (2014) Migrating cells mediate long-range WNT signaling. Development 141 (10):2057-2063. doi:10.1242/dev.107656

37. Momose T, Tonegawa A, Takeuchi J, Ogawa H, Umesono K, Yasuda K (1999) Efficient targeting of gene expression in chick embryos by microelectroporation. Dev Growth Differ 41 (3):335-344

38. Yasuda K, Momose T, Takahashi Y (2000) Applications of microelectroporation for studies of chick embryogenesis. Dev Growth Differ 42 (3):203-206

39. Rueden CT, Schindelin J, Hiner MC, DeZonia BE, Walter AE, Arena ET, Eliceiri KW (2017) ImageJ2: ImageJ for the next generation of scientific image data. BMC bioinformatics 18 (1):529. doi:10.1186/s12859-017-1934-z

40. Schindelin J, Arganda-Carreras I, Frise E, Kaynig V, Longair M, Pietzsch T, Preibisch S, Rueden C, Saalfeld S, Schmid B, Tinevez JY, White DJ, Hartenstein V, Eliceiri K, Tomancak P, Cardona A (2012) Fiji: an open-source platform for biologicalimage analysis. Nature methods 9 (7):676-682. doi:10.1038/nmeth.2019 41. Linkert M, Rueden CT, Allan C, Burel JM, Moore W, Patterson A, Loranger B, Moore J, Neves C, Macdonald D, Tarkowska A, Sticco C, Hill E, Rossner M, Eliceiri KW, Swedlow JR (2010) Metadata matters: access to image data in the real world. J Cell Biol 189 (5):777-782. doi:10.1083/jcb.201004104 
42. Thevenaz P, Ruttimann UE, Unser M (1998) A pyramid approach to subpixel registration based on intensity. IEEE transactions on image processing : a publication of the IEEE Signal Processing Society 7 (1):27-41. doi:10.1109/83.650848

43. Preibisch S, Saalfeld S, Tomancak P (2009) Globally optimal stitching of tiled 3D microscopic image acquisitions. Bioinformatics (Oxford, England) 25 (11):1463-1465. doi:10.1093/bioinformatics/btp184

\section{FIGURE LEGENDS}

FIGURE 1. Chick secondary neurulation (SN). (A) Drawing of a HH15 chick embryo showing the caudal region where $\mathrm{SN}$ is taking place. The progression of SN can be followed along the antero-posterior axis of the same embryo. (B) Dorsal view of the boxed region in A showing the distribution of actin (Phalloidin) in white. The cells in posterior regions are mesenchymal cells (MCs), those in the intermediate regions are undergoing the mesenchymal-to-epithelial transition (MET) and the anterior cells are neuroepithelial cells (NECs). The transverse sections in C-G correspond to different levels along the antero-posterior axis. Scale bar $=40 \mu \mathrm{m}(\mathrm{C}-\mathrm{G})$ Transverse sections at different antero-posterior levels showing the distribution of actin. Scale bar $=40 \mu \mathrm{m}$ (C'-G') Schematic representation of chick SN showing major cell and tissue rearrangements. Neural progenitors are shown in light blue, the surrounding mesoderm is in brown and the notochord appears in dark blue. (C'-D') Chick SN starts with the convergence of neuro-mesodermal progenitors in the centre of the tissue and the formation of a solid medullary chord (grey arrows). (E') Cells located dorsally and at the periphery of the medullary chord are the first to undergo the MET. Epithelialization propagates ventrally, although the cells in the centre of the tissue remain mesenchymal and small lumens open up between the peripheral epithelial and central mesenchymal cell populations. (F') The small cavities formed coalesce in a dorsoventral gradient to 
form a single central lumen and the mesenchymal cells that remain in the centre are finally cleared from the lumen. (G') The result of this MET and central clearing process is that a hollow neural tube is formed that is surrounded by neuroepithelial cells.

FIGURE 2. Chick in ovo electroporation of SN cells. (A) The DNA mix (green) is injected into the concave region of the epiblast at the caudal end of a HH9 chick embryo. The positioning of the electrodes is shown, whereby the positive platinum electrode is placed below the embryo through a hole made in the vitelline membrane, while the tungsten needle connected to the negative electrode is positioned on top of the embryo. Both electrodes are positioned parallel to the antero-posterior axis of the embryo. (B) Scheme of a transverse section of a chick embryo at the level of the dotted line in A. The epiblast is electroporated by applying the current from top to bottom (red arrow). (C) Image of the two electrodes used. (D) Schematic representation of a HH15 chick embryo showing the region electroporated using our method 24 hours-postelectroporation (hpe). (E) Dorsal view of the boxed region in D following the electroporation of pSox 2:eGFP. Both the caudal neural tube and the $\mathrm{SN}$ region are efficiently electroporated. Scale bar $=200 \mu \mathrm{m}$. (F-G) Transverse sections at the two levels indicated in D showing the electroporation of TopFlash:d2eGFP. F is at the level where the lumen is forming, and where both peripheral epithelial (red arrows) and central mesenchymal cells (orange arrow) are labelled. G shows the efficient electroporation of the posterior progenitor cells. Scale bar $=40 \mu \mathrm{m}$.

FIGURE 3. Embryo culture for in vivo time-lapse imaging. (A) Paper rings are prepared by making a clover leaf hole in the centre of $2 \times 2 \mathrm{~cm}$ squares of Whatman grade 1 filter paper with a paper punch and cutting off the corners. (B) The paper ring is laid over the embryo inside the egg, so that the embryo is positioned in the centre. (C) After carefully cutting out the embryo attached to the paper ring, it is then transferred 
dorsal side up into an imaging plate containing Albumen/Agar. (D) Selected embryos (up to 6) are then transferred to the culture chamber for imaging. (E) The culture chamber is finally sealed with insulation tape. Scale bar $=0.5 \mathrm{~cm}$.

FIGURE 4. Normal chick embryo development. Frames from a 5x WT video showing normal chick embryo tail bud elongation and blood vessel formation (hh:mm) (see Movie S1). The embryo was at HH13 when the video begins and it develops to HH16. Scale bar $=500 \mu \mathrm{m}$.

FIGURE 5. In vivo analysis of SN cells. (A) First frame from a 20x video showing a pSox2:eGFP electroporated embryo (see Movie S2). The somites, neural tube, SN region and tailbud are indicated. (B) Frames of the same movie as development proceeds, with the tailbud growing caudally and SN advancing. (C) Tracks obtained from the embryo in A-B using the Manual Tracking plug-in of the ImageJ software (see Movie S3). (D) Frames from the video in A-B at a higher magnification. Single cells are easily followed over time. The cells enclosed by dotted lines correspond to the light blue and magenta tracks in $\mathrm{C}$. The blue cell divides and generates two daughter cells. Scale bars $=50 \mu \mathrm{m}$.

MOVIE S1. In vivo time-lapse imaging of a WT chick embryo (5x) over 10h showing normal tail bud elongation and blood vessel formation (hh:mm). Related to Figure 4. MOVIE S2. In vivo time-lapse imaging of a pSox 2:eGFP electroporated embryo (20x) over 10h. Related to Figure 5.

MOVIE S3. In vivo time-lapse imaging of a pSox 2:eGFP electroporated embryo showing the tracks obtained using the Manual Tracking plug-in of the ImageJ software. Related to Figure 5. 